\section{A unique critique}

Review articles shape the way many chemists perceive the state of the art. Accordingly, there is great responsibility on authors, referees and editors to get things right. At Nature Reviews Chemistry, we rely on reports from discerning and diverse (in every sense of the word) referees to make informed decisions about the content we offer our readers each month. Reviewing a primary research manuscript is largely a matter of evaluating if new conclusions are well-founded and, if they are, whether or not they are significant advances ${ }^{1}$. These two criteria also apply to refereeing review manuscripts, which must additionally be subject to a stringent critique in terms of clarity.

So who does all this critiquing? Well, editors often make a general recommendation, but beyond that, we look to reviewers from the research community to dissect a manuscript. The way we choose reviewers is similar to the way we choose authors: we seek out leading scientists actively working in one or all of the topic areas being described. Sometimes we look for reviewers working on slightly different topics to those being described, particularly when authors discuss interdisciplinary science. It's only human not to know technical aspects of every topic, and if something in a manuscript is unclear to an intelligent non-expert reader, then the problem is likely with the manuscript rather than the reader. For example, if a reviewer is not a specialist on a topic, they serve as a good model for the intelligent non-experts who will later read the review as, perhaps, an introduction into a research area. Rest assured, if we tap someone on the shoulder to ask if they will review a manuscript, we've chosen them with good reason.

Having appointed a crack team of reviewers, what do editors look for in each report? The most obvious answer is a detailed technical assessment of the manuscript. It's true that editors of any journal will need such advice to make a prudent decision about whether or not to go forward with a submission. To this end, it helps us to get to know each reviewer ${ }^{2}$. Do they all know every aspect backwards, or is she perhaps a theoretician and he an experimentalist? By introducing themselves, reviewers give editors an idea of which sections they critiqued from a technical perspective and which ones were assessed more in terms of presentation. Yes, assessing scholarly presentation - and we don't mean just spotting typos ('that hyphen really should be an en dash') - is an important part of assessing a review manuscript. Indeed, presentation extends beyond cosmetics, such as fancy figures and pretty prose, to include clarity and logical flow. We are always taught to write with the reader in mind, and indeed the best reviewers make themselves familiar with a journal's content and write their reports with empathy for its readership. Thus, given that we aim to interest experts and non-experts alike, it's particularly telling when an expert reviewer tells us that certain aspects may not be comprehensible to a prototypical non-expert reader.

Our articles are relatively succinct and authors have to be selective about which topics they describe. Here, editors count on reviewers to assess whether or not the content is balanced and representative of the manuscript's scope. This pertains to the choice of examples cited ('this manuscript should be rejected unless the authors cite my entire back-catalogue'), as well as to recognizing multiple points of view on a controversial topic. Some of our articles may offer more conjecture and/or a personal tone, but this should never come at the expense of objectivity. Reviews perhaps don't need to include the 'hard-sell' of a field that primary research papers do, so undue bias in a review is particularly inexcusable.

Describing new conclusions is just as important for Nature Reviews Chemistry articles as it is for the best research articles. We expect all of our offerings to include new insights that raise the eyebrows of even the most seasoned researchers of a given topic. If you are the chemistry font of knowledge we chose to critique a manuscript for us then, chances are, you probably will not learn as much from it as a non-expert would. Nevertheless, we'd hope that you still find something new. Failing that, by all means don't hold back in your report, but being constructive and timely in your criticism goes a long way. Remember the golden rule of reviewing: review for others as you would have others review for you ${ }^{3}$.

All told, reviewing a review is a great responsibility because these articles spell out unsolved problems to specialists and welcome new scientists to a field. Each day at Nature Reviews Chemistry, we have the privilege of witnessing how, through the peer-review process, manuscripts evolve into papers that enlighten our community. This privilege is made possible by the careful and selfless work of our reviewers, to whom we are gratefully indebted.

\footnotetext{
The perfect peer [Editorial]. Nat. Chem. 3, 831 (2011).

Stiller-Reeve, M. How to write a thorough peer review. Nature. https://doi.org/10.1038/d41586-018-06991-0 (2018).

3. McPeek, M. A. et al. The golden rule of reviewing. Am. Naturalist 173, E155-E158 (2009).
} 